\title{
Competenze, prassi e legittimità profetica del Dante dictator illustris
}

\section{Elementi di un’interpretazione sociologico-retorica delle epistole}

\begin{abstract}
Nel saggio si fornisce un'interpretazione complessiva delle 12 lettere dantesche. Si definiscono in prima istanze le competenze di Dante nel dominio del dictamen, partendo dal testo del Dve incentrato sui gradi della «constructio». Si verifica, poi, la corrispondenza tra la teoria retorica espressa nel capitolo e la prassi di scrittura delle epistole. A partire da questo quadro, si propone un confronto con altri autori volgari della generazione precedente che hanno riservato al dictamen un ruolo centrale (Guittone d’Arezzo e Brunetto Latini). In seguito, si analizza la scrittura dantesca da un punto di vista strettamente retorico, comparando lo stile di Dante con lo stilus curie romane, che sembra il più vicino. Si analizza, infine, il processo di auto-investitura profetica di Dante, che nelle lettere rende visibile il ruolo che il profetismo assume per fornire a Dante, esule e laico ormai privo di ruoli istituzionali riconosciuti, una legittimazione di natura carismatica.
\end{abstract}

The article proposes a comprehensive interpretation of Dante's 12 epistles. I first define Dante's competence in the field of ars dictaminis, relying on the passage in De vulgari where he discusses the degrees of «constructio». I then proceed to verify the correspondence between said rhetorical theory and the writing practice developed in his epistles. In this framework, I compare Dante with other authors from the previous generation who likewise wrote in the vernacular and accorded great importance to the dictamen (Guittone d'Arezzo and Brunetto Latini). Then, I analyze Dante's writing from a rhetorical point of view and compare his style with the stilus curie romane, which seems to be the closest to his.

Annotazione: Ho discusso parti di questo saggio con Elisa Brilli, Maria Conte, Fulvio Delle Donne, Benoît Grévin, Domenico Losappio, Giuliano Milani, Marco Petoletti, Sylvain Piron, Gian Luca Potestà, Justin Steinberg: le loro letture mi hanno permesso di mettere a fuoco numerose questioni che ho provato ad affrontare ed approfondire, e per questo li ringrazio. Resta inteso che limiti ed errori del presente articolo sono integralmente a carico dello scrivente.

Le citazioni del testo dantesco provengono dalle edizioni esplicitamente citate; ho invece talvolta ritoccato la traduzione.

Antonio Montefusco, Università Ca’ Foscari Venezia

Ә Open Access. (c) 2020 Antonio Montefusco, published by De Gruyter. (c) BY This work is licensed under the Creative Commons Attribution 4.0 International License (CC BY 4.0).

https://doi.org/10.1515/9783110590661-006 
Finally, I examine Dante's prophetic self-investiture and its role in providing Dante, exiled layman without a true institutional role, with a charismatic legitimization.

Parole chiave: Dante, ars dictaminis, Guido Faba, Tommaso di Capua, cursus, profetismo.

\section{Un’idea di dictamen}

In Dve II, vi, 2-4 è percepibile con immediatezza un'intensificazione tecnica dell'argomentazione che vira decisamente verso l'ars dictaminis. L'inserzione di una lista di sintagmi in prosa latina, organizzati secondo una gerarchia ascendente su diversi piani (sintattico, stilistico, metaforico) e inquadrati in un contesto spiccatamente scolastico-pedagogico, ${ }^{1}$ è infatti intesa a spiegare quale sia il grado di «constructio» (“costruzione”) dotato di massima eleganza («urbanitas plenissima»), specificata, poi, come grado di costruzione "saporito, venerabile ed eccellente” («sapidus et venustus etiam et excelsus») nonché "il più eccellente” («excellentissimus»). ${ }^{2} \mathrm{Nel}$ secondo libro dell'incompiuto trattato latino, Dante ha stabilito ordinatamente che al volgare illustre si addicono solo un certo tipo di versificatori, trascelti sulla base del principio della "convenentia», ${ }^{3}$ quindi tre tematiche («salus, venus, virtus») specifiche, ${ }^{4}$ un metro particolare (la canzone) ${ }^{5}$ e uno stile preciso (quello tragico), ${ }^{6}$ surrogato da capacità tecni-

1 Cfr. dopo, a nota 11.

2 «Est enim sciendum quod constructionem vocamus regulatam compaginem dictionum; ut Aristotiles phylosophatus est tempore Alexandri. Sunt enim quinque hic dictiones compacte regulariter, et unam faciunt constructionem. Circa hanc quidem prius considerandum est quod constructionum alia congrua est, alia vero incongrua. Et quia, si primordium bene discretionis nostre recolimus, sola supprema venamur, nullum in nostra venatione locum habet incongrua, quia nec inferiorem gradum bonitatis promeruit. (...) Est ut videtur congrua quam sectamur. Sed non minoris difficultatis accedit discretio priusquam quam querimus attingamus, videlicet urbanitate plenissimam» (Dve II, vi, 2-4).

3 È il tema di Dve II, i, 8: «quapropter, si non omnibus competit, non omnes ipsum debent uti, quia inconvenienter agere nullus debet».

4 "Quare hec tria, salus videlicet, venus et virtus, apparent esse illa magnalia que sint maxime pertractanda, hoc est ea que maxime sunt ad ista, ut armorum probitas, amoris accensio, et directio voluntatis» (Dve II, ii, 8).

5 «Horum autem modorum cantionum modum excellentissimum esse pensamus; quare, si excellentissima excellentissimis digna sunt, ut superius est probatum, illa que excellentissimo sunt digna vulgari, modo excellentissimo digna sunt, et per consequens in cantionibus pertractanda» (Dve II, iii, 3).

6 «Quare, si bene recolimus summa summis esse digna iam fuit probatum, et iste quem tragicum appellamus summus videtur esse stilorum, illa que summe canenda distinximus isto solo 
che e cultura classica, ${ }^{7}$ e infine un verso proprio, il superbissimum carmen dell'endecasillabo. ${ }^{8}$ Per quanto, dunque, nei primi cinque capitoli si possa vedere una compatta gradazione dell'argomentazione, ${ }^{9}$ è altrettanto evidente che Dante segnali al lettore che, col capitolo VI, si affronta un tema importante in maniera innovativa. ${ }^{10}$

Dopo una ricapitolazione di quanto già detto fino a lì, ${ }^{11}$ Dante fornisce una precisa definizione del concetto di "costruzione” come «regulat $a$ compago dictionum», cioè come “connessione di parole sottoposte a una regola”; segue immediatamente un'esemplificazione, consistente in un periodo latino composto di cinque parole «compacte regulariter» - si varia qui, con gioco retorico, la definizione della costruzione come «regulat $a$ compago», e questo virtuosismo serve all'autore per dare già il tono dell'argomentazione e individuare una tradizione argomentativa precisa, nonché per ritagliarsi un possibile pubblico di riferimento. ${ }^{12}$ L'esempio in questione è «Aristotiles phylosophatus est tempore Ale-

sunt stilo canenda, videlficet salus, amor et virtus, et que propter ea concipimus, dum nullo accidente vilescant» (Dve II, iv, 8).

7 «Sed cautionem atque discretionem habere sicut decet, hic opus et labor est, quoniam nunquam sine strenuitate ingenii et artis assiduitate scientiarumque habitu fieri potest (...). Et ideo confutetur eorum stultitia, qui, arte scientiaque immunes, de solo ingenio confidentes, ad summa summe canenda prorumpunt; et a tanta presumptuositate desistant; et si anseres natura vel desidia sunt, nolint astripetam aquilam imitari» (Dve II, iv, 9-10).

8 «Quorum omnium endecasillabum videtur esse superbius, tam temporis occupatione, quam capacitate sententie, constructionis, et vocabulorum; quorum omnium specimen magis multiplicatur in illo, ut manifeste apparet; nam ubicunque ponderosa multiplicantur, multiplicatur et pondus» (Dve II, v, 3).

9 Si veda la gradazione negli incipit dei vari capitula, scandita da particelle temporali: «postquam» (Dve II, ii,1); «nunc autem» (II, iii, 1); «quando (...) antequam» (II, iv, 1); «satis dixisse videmur» (II, v, 1); segue, nel VI, una rottura evidente, con uso di una formula «versatur intentio» (II, vi, 1), estratta, secondo Mengaldo, dal «linguaggio filosofico ab antiquo» (Dve [Mengaldo]), formula qui riferita al subiectum generale del trattato: «quia circa vulgare illustre nostra versatur intentio».

10 Su questo capitolo, si raccomanda in particolare l'ampio commento di Tavoni, Dve (Tavoni), pp. 1434-1445, che ricorre sistematicamente, e a giusto titolo, alla tradizione dell'ars dictaminis, raccogliendo una linea di commento al testo che risale a Marigo, e che si è quindi precisata ulteriormente con Mengaldo; ampio spazio a questa tradizione è anche nel commento di Fenzi.

11 «Quia circa vulgare illustre nostra versatur intentio, quod nobilissimum est aliorum, et ea que digna sunt illo cantari discrevimus, que tria nobilissima sunt, ut superius est astructum, et modum cantionarium selegimus illis tanquam aliorum modorum summum, et ut ipsum perfectius edocere possimus quedam iam preparavimus, stilum videlicet atque carmen, nunc de constructione agamus» (Dve II, vi, 1).

12 Sull'interpretazione di questo capitolo è riferimento l'articolo di Tavoni, Chersoni, Ipotesi d'interpretazione, soprattutto le pp. 131-142 (il resto del saggio è dedicato all'analisi sintattica 
xandri», cioè "Aristotele filosofò al tempo di Alessandro”. A voler specificare, il periodo è strutturato in maniera basica, perché l'ordine delle «dictiones» è organizzata secondo l'ordine naturale, seguendo cioè la progressione soggetto + verbo + complemento; ${ }^{13}$ la «clausula», il sintagma che chiude il periodo (in questo caso «tèmpore Àlexàndri»), vede succedersi una parola proparossitona e un quadrisillabo parossitono, secondo una scansione che veniva chiamata cursus velox e che costituiva la cadenza ritmica più ricercata (se non obbligatoria) davanti alle pause forti. ${ }^{14}$ Tale tipo di costruzione, per quanto semplice, rientra nella categoria delle costruzioni "congrue" o "corrette", le sole che l'autore ha intenzione di analizzare ed esemplificare a scopo precettivo per la pratica letteraria dei poeti illustri.

comparata delle 11 canzoni esemplari della «supprema constructio»); nell'articolo è mobilitata e ripresa anche l'intera bibliografia pregressa sul rapporto tra l'argomentazione dantesca qui sviluppata e le tradizioni retorica e grammaticale precedenti e coeve: ciò mi esime dal richiamarla a mia volta. Su compago, cfr. p. 132; si aggiungano gli interessanti rilievi di Fenzi, che, al classico Prisciano (e dunque alla tradizione specificamente grammaticale), aggiunge Albertano, Liber II, 63 (con riferimento alla compagine sociale), esempio pregnante data la continua oscillazione di Albertano da Brescia tra retorica e pragmatica (su cui cfr. almeno Artifoni, Prudenza del consigliare).

13 Alle osservazioni di Marigo, che afferma che l'esempio è «una proposizione in cui le parole sono collocate nell'ordine naturale e, coll'accordo sintattico del genere, numero e caso, compacte regulariter» (Dve [Marigo], ad locum) e di Mengaldo («l'ordine è quello naturale soggettoverbo-complemento [...] ma è già presente la clausola ritmica [velox] e i nomi propri sono di personaggi di nobile estrazione storica, come nella formulistica d'intenzione più elevata, non Pietri e Berte qualsiasi», Dve [Marigo], ad locum), Tavoni, Chersoni, Ipotesi d'interpretazione, p. 132, aggiungono Matteo di Vendôme, Ars versificatoria (Faral, Les arts poétiques, p. 118) con riferimento all'importanza di preservare l'ordine naturale per non danneggiare il senso.

14 Sul cursus nella prosa medievale, sono ancora importanti i volumi di Lindholm, Studien zum Mittellateinischen Prosarhythmus e Janson, Prose Rhythm, su cui bisogna tenere presente la recensione di Orlandi, pp. 701-718; una rivalutazione complessiva del ruolo del cursus nella prosa medievale è in Grévin, L'empire d'une forme, pp. 857-881; sulla terminologia medievale, diversa da quella adottata nella tradizione degli studi contemporanei (cfr. anche dopo), cfr. Turcan-Verkerk, La théorisation progressive, pp. 179-201. Lo studio dell'uso del cursus in Dante è oggetto di una ampia tradizione di studi: si ricordano, in particolare, quelli di Parodi, Intorno al testo; Di Capua, Appunti sul “cursus” e le pp. riunite anche in Di Capua, Fonti ed esempi; Rajna, Per il "cursus" (non solo sulle lettere) come anche Mengaldo, Cursus, e Mikkel, Cursus in Dante. Già in Parodi e Di Capua il cursus ha permesso di proporre aggiustamenti al testo tràdito: sulle conseguenze filologiche di tale approccio (con esempi danteschi), si veda Chiesa, L'impiego del cursus. Una prima sistemazione delle clausole ritmiche nelle epistole è in Rossetto, L'uso del “cursus”, e un'interpretazione complessiva in Di Patre, Un cursus geometrico?. Si veda ora, però, l'articolo di B. Grévin, Le Epistole dantesche in questo volume, che è anticipazione di una monografia sulla questione; si tengano presente anche le osservazioni nel saggio di F. Delle Donne, L'epistola II, sempre in questo volume, che propone un approccio leggermente diverso. 
In questo caso, però, l'esemplificazione è realizzata con periodi prosastici in latino. Conviene riportarla:

sed non minoris difficultatis accedit discretio prius quam, quam querimus, actingamus, videlicet urbanitate plenissimam. Sunt etenim gradus constructionum quamplures: videlicet insipidus, qui est rudium; ut Petrus amat multum dominam Bertam. Est et pure sapidus, qui est rigidorum scolarium vel magistrorum, ut Piget me, cunctis pietate maiorem, quicunque in exilio tabescentes patriam tantum sompniando revisunt. Est et sapidus et venustus, qui est quorundam superficietenus rethoricam aurientium, ut Laudabilis discretio marchionis Estensis et sua magnificentia preparata cunctis, cunctis illum facit esse dilectum. Est et sapidus et venustus etiam et excelsus, qui est dictatorum illustrium, ut Eiecta maxima parte florum de sinu tuo, Florentia, nequicquam Trinacriam Totila secundus adivit. Hunc gradum constructionis excellentissimum nominamus, et hic est quem querimus, cum suprema venemur, ut dictum est (Dve II, vi, 4-5).

L'esempio eccellente, che è sempre il grado più elevato verso cui tende l'argomentazione precettiva dantesca, è qui definito come tipico dei «dictatores illustres», ovverosia dei “dettatori”, i grandi prosatori e, in particolare, autori di epistole, la cui scrittura era oggetto di trattazione sistematica nei manuali di ars dictaminis. ${ }^{15}$ A ragione, dunque, uno dei grandi commentatori del trattato dantesco ha affermato che qui «alla prospettiva grammaticale viene subito cumulata quella retorica»: ${ }^{16}$ si traduca l'espressione pensando che, per la esatta definizione di «constructio», Dante ha attinto dall'insegnamento grammaticale, fondato essenzialmente sui testi tardo-antichi di Donato e di Prisciano e incentrato sulle parti del discorso, mentre, laddove si passa a mostrare i gradi dei diversi costrutti, utilizza la strumentazione tecnica del dictamen, secondo la seguente ascensione: 1 . correttezza grammaticale; 2 . ricercatezza nell'ordine delle parole (mediante il ricorso all'ordo artificialis) e uso del cursus; 3. aggiunta di figure di pensiero (i «colores») adatte a uno stile mediocre (ornatus facilis); 4. impiego della metafora (transumptio) insieme alle altre figure retoriche tipiche dello stile tragico (enfasi, personificazione etc.), classificate come ornatus difficilis. ${ }^{17}$

15 Sul ruolo delle Summae e la loro diffusione, cfr. almeno Grévin, Rhétorique du pouvoir e l'introduzione di Delle Donne a Nicola da Rocca, Epistole; sull'ars dictaminis in generale, in attesa del manuale Ars dictaminis in corso di stampa, si può ricorrere con profitto a Delle Donne, Santi, Dall'ars dictaminis, e Grévin, Turcan-Verkerk, Le dictamen, che è dotato di un'amplissima bibliografia (e che ci esime di specificare altrimenti).

16 Dve (Mengaldo), ad locum.

$17 \mathrm{Si}$ riassume qui un dibattito che è stato piuttosto ampio, e con notevoli addentellati, su questo capitolo del Dve. Si prendano come particolarmente esemplificativi gli interventi di Scaglione, Dante and the Rhetorical, che ha sottolineato l'«idiosyncratic way» in cui Dante avrebbe mescolato il pensiero strettamente grammaticale (e più specificamente sintattico) della tradizione grammaticale con quello retorico della tradizione del dictamen, e di Corti, Dante, 
Questa modellizzazione è per noi particolarmente preziosa, perché ci informa con estrema precisione delle competenze nel dominio del dictamen - come si è detto, una specifica e disciplinare parte della retorica nel medioevo maturo - che sono certificabili per l'Alighieri alla data del 1304-1307 (presunti termini post e ante per la redazione del trattato incompiuto). ${ }^{18} \mathrm{Si}$ tratta dello sfondo tecnico su cui è costruita la prassi epistolare di Dante, e per questo merita ancora qualche indugio, sia per chiarire la posizione dantesca in merito alla cultura dictaminale del suo tempo, sia per poter analizzare le poche lettere (12 in tutto: teniamo da parte quella a Cangrande, ancora controversa $)^{19}$ sicuramente attribuibili alla sua mano. Sarà questo l'oggetto delle prossime pagine (§ 2-4), base di un ulteriore ragionamento che si propone di rivalutare le strategie di legittimazione utilizzate da Dante nella scrittura epistolare nella fase finale della produzione epistolare (con le arrighiane e la lettera ai cardinali, 1311-1314), laddove l'assenza di un rapporto formalizzato con un potere istituzionalizzato lo induce a rafforzare l'autocoscienza profetica (§ 5).

\section{Tra Bologna, Arezzo e Firenze}

Bisogna sottolineare, innanzitutto, che il paragrafo del Dve sopra riportato condensa in un numero limitato di esempî una materia che i manuali di dictamen svolgevano con grande dispendio di indicazioni puntuali, divise in sezioni differenti. ${ }^{20}$ Per comprendere questo sforzo di sintesi, basti pensare che, tra i quattro

che ha individuato nella teorizzazione modista una fonte anche per la supprema constructio dantesca. Una valutazione equilibrata della questione, che tende a valorizzare l'apporto della tradizione retorica anche sulla teorizzazione sintattica elaborata in questo passo del $D v e$, è in Tavoni, Chersoni, Ipotesi d'interpretazione, pp.137-142 (ma si veda già il commento di Dve [Tavoni], pp. 1435-1436).

$18 \mathrm{E}$ - si noti - limiti temporali anche coincidenti ad annum con le epistole concretamente conservate, collocandosi la più antica probabilmente al 1304 (Ep I); per la retrodatazione della II, proposta da Indizio, Sul mittente, seguito, tra gli altri, anche da me (Montefusco, Un approccio al corpus, p. 453), cfr. ora, in questo volume, il saggio di Tavoni, Le epistole I e II nella vita di Dante (fatti, personaggi, date, testualità, ideologia), che ripropone, con argomenti convincenti, l'ordine cronologico proposto dalla edizione nazionale.

19 Sulle differenze stilistiche tra l'epistola a Cangrande e il resto del corpus (e le altre opere dantesche), si vedano ancora con profitto le pagine di Dronke, Dante e le tradizioni. Sulla problematicità dell'epistola XIII, oltre al corposo commento procurato da L. Azzetta, sostenitore della sua autenticità, cfr. da ultimo Inglese, Dante (?) a Cangrande.

20 Contrariamente a Ad Herenn. dove la trattazione è più conseguente e compatta: cfr. IV, 1012; cfr. invece almeno Bene da Firenze, Candelabrum, che riprende il problema riorganizzandolo su I, 8 e II, 69 (con riprese anche da Trasmondo e in rapporto con Bernardo da Bologna). 
esempî riportati, i primi due sembrano da leggersi piuttosto come dictamina imperfetti, in cui, cioè, permangono dei vitia di costruzione: nel primo è evidente la tematica triviale presa nel suo senso proprio, mentre nel secondo emerge una certa obscuritas (è il termine tecnico nelle artes) ${ }^{21}$ dovuto all'omissione di membri indispensabili per la comprensione (per es., un eorum dopo quicunque), che però avrebbero danneggiato il rigido isosillabismo (Dante lo definisce "degli scolari") ricercato per la frase. "Vitiis itaque penitus eiectis», direbbe un maestro di dictamen; ${ }^{22}$ Dante snocciola due periodi che invece rispondono alle esigenze del dettatore più, e poi ancor più, illustre. Se da una parte, questa sinteticità sembra ritagliare un pubblico preciso per il trattato, capace, con questi pochi scorci, di individuare le indicazioni d'insieme, dall'altra la terminologia usata da Dante nonché l'attitudine complessiva, orienta su un corpus testuale preciso: mi riferisco al dictamen come veniva insegnato a Bologna, e in particolare a quello ampiamente rinnovato all'inizio del Duecento. ${ }^{23}$

Rispetto alle fonti solitamente invocate in questo specifico ambito, il "criptomanuale" tratteggiato da Dante sembra prossimo alle opere del maestro bolognese Guido Faba, attivo negli anni '20-'40 del XIII secolo, e la cui influenza fu determinante su scala europea. ${ }^{24}$ L'aggettivazione con cui l'Alighieri produce la sua gradazione, incentrata sulla conquista di una venustas ("grazia") che ha una base gustativa ("saporito"), a fondamento della scala di giudizio delle varie costruzioni (insipidus - pure sapidus - sapidus et venustus - sapidus, venustus et excellentissumus), è con tutta evidenza debitrice della dulcedo rethorice venustatis (legata alla dulcedo saporis) che è uno degli elementi del perfetto dictamen nelle opere del Faba, e in particolare nel suo manuale più fortunato, intito-

21 Cfr. almeno Bene da Firenze, Candelabrum, I, xi, 10-1 (e per le conseguenze sulla scrittura letteraria del Due-Trecento, cfr. Montefusco, Bischetti, Prime osservazioni, pp. 202-205). In questo volume, il saggio di Tomazzoli, Funzioni delle metafore, dimostra come nelle epistole politiche Dante metta in pratica una retorica dell'explanatio, allontanando il rischio di obscuritas. 22 «Vitiis itaque penitus eiectis, in summa teneas quod omne dictamen commodum et perfectum tria requirit: bonam gramaticam, perfectum sensum locutionis, et verborum ornatum. $\mathrm{Si}$ autem hec tria dictator fecerit, perfecte dictabit» Guido Faba, Summa dictaminis, p. 295.

23 «I tre esempi di costruzione sintattico-retorica forgiati da Dante in II, vi, 4 (...) sono così evidentemente fatti su misura per un pubblico di cultori di artes dictandi (tanto più data la singolarissima scelta di dare, come modelli di sintassi poetica volgare, tre esempi di sintassi prosastica latina), da orientare decisamente su Bologna, capitale delle artes dictandi» (Tavoni, Qualche idea, p. 103, ma si vedano le pp. 97-103, che riassumono i dati raccolti nel commento citato). Personalmente, considero l'ipotesi plausibile; si tenga tuttavia presente anche la ricostruzione differente di Inglese, Vita di Dante, p. 521; in disaccordo è anche Fenzi nel suo commento al Dve: Dve (Fenzi), pp. XXIII-XXIV.

24 Una prima ricognizione della tradizione manoscritta è in Pini, La tradizione manoscritta. 
lato tradizionalmente Summa dictaminis (ma che sarebbe più corretto chiamare Ars), sunto della sua attività. ${ }^{25}$

Redatta in risposta alla pubblica lettura della Rethorica antiqua del maestro anticiceroniano Boncompagno da Signa (1170 ca. - post 1240), ${ }^{26}$ l'Ars di Guido ne condivide l'orgoglio corporativo, che indica nel dictamen un sapere sacrale, custodito dal magister che lo dispensa ai proprî allievi, aprendo agli intellettuali l'ordine dell'universo (la cosiddetta machina mundialis). Il Dante dictator del De vulgari presenta una piena consonanza con questo orgoglio, che, nel XIV secolo, è diventato piuttosto diffuso nella manualistica, in particolare in una serie di prologhi di carattere autobiografico che accompagnano e ribadiscono l'ascesa della tecnica del dictamen come "sapere egemonico" (secondo la nota definizione di Benoît Grévin). ${ }^{27}$ Le rivendicazioni di originalità, pure così diffuse nell'opera omnia dantesca, dalla Vita Nova alla Commedia, nel De vulgari eloquentia assumono una curvatura che risente di questa particolare stagione di auto-promozione, sociale e culturale insieme, dei grandi maestri di dictamen dell'inizio del Duecento. Nella prima di queste rivendicazioni, ad apertura del trattato, si afferma la volontà di «discretionem aliqualiter lucidare illorum qui tanquam ceci ambulant per plateas» (Dve I, i,1), ${ }^{28}$ riprendendo alla lettera uno

25 L'espressione ritorna in più luoghi del testo (Guido Faba, Summa, pp. 289 e 295) e deriva dalla definizione del celebre prologo, dove il sapere retorico posseduto dal magister, rappresentato come un viridarium, è caratterizzato da dolcezza di canto, bellezza e profumo: «advenite nunc omnes ad viridarium magistri Guidonis, qui dona sophie cupitis invenire, ubi dulces avium cantus resonant et suaviter murmurant a fontibus rivuli descendentes, flores similiter apparent vernantes et lilia venustatis, rose quoque specioso consurgunt, et cynnamomum et balsamum ac viole non desinunt redolere» (p. 287). Un intreccio tra dolcezza e sapore è anche all'inizio del testo, proprio in seguito rispetto al prologo, laddove si introduce al tema del buon dictamen: «quia scire malum non est malum, sed peccatum est potius operari; cum huius rei peritiam habere debeat unusquisque, ut que sint nocitura, docente notitia, fugiat, quod aliter numquam posset, et faciat, inspirante Domino, que sint iusta; ad modum serentis qui terram spinis ac tribulis purgat ut fructus mundus appareat et sincerus, ad similitudinem illius qui studiose vas lavat, ut eiecta sorde antiqui vitii ad novi saporis dulcedinem preparetur; iterum eo perspecto quodvirtutes plantari non valent, nisi ea que sunt contraria extirpentur; priori facie vitiis estirpatis de regulis subsequenter tractemus prosaici dictaminis et doctrina» (p. 288). Significativa anche la presenza del dictator «insipidus» in Jacques De Dinant, Summa, rr. 71-73, in Polak, A textual study, p. 63 (segnalatomi da Domenico Losappio, che ringrazio), che permette di introdurre la pista parallela delle poetriae.

26 Secondo la ricostruzione di Gaudenzi, Sulla cronologia, p. 183; per la biografia di Faba, cfr. però anche il classico Kantorowicz, An "Autobiography", e Saiani, La figura di Guido Faba.

27 Questi prologhi e il loro significato nel contesto della retorica duecentesca è studiato, in parte sulla scia di Kantorowicz, An "Autobiography", in Artifoni, "Sapientia Salomonis".

28 Ma andrà tenuta presente anche una forte tendenza didattica insita nell'uso del «lucidare», come rilevato da Fenzi che rimanda, tra gli altri, al Candelabrum di Bene e in specie al suo incipit (Dve [Fenzi], ad locum), e se ne raccomanda, a questo proposito, anche il commento di 
dei topoi di questa stagione: il sapere retorico che supera le tenebre, cristallizzato fin nel titolo del testo di Bene da Firenze (Candelabrum, appunto) nonché nella solita Ars del Faba, dove l'oscurità è ricacciata allorquando si bussa alla porta del dictamen («tenebris relegatis, ianua dictaminum sit pulsantibus aperta»). ${ }^{29}$

Nel secondo libro del trattato Dante passa a occuparsi, in maniera praticoprecettiva, del genere lirico più illustre (la canzone), dopo una discussione degli elementi che ne permettono la realizzazione: quale tipo di volgare, quale ordine di versificatori, quali argomenti e quale stile, la misura dei versi e finalmente la constructio (Dve II, i-vi). In questo contesto così tecnicizzato si infittiscono i passaggi che stigmatizzano gli errori del passato; il primo è collocato nella discussione della “materia” e si appoggia sui versi dell'Ars poetica «sumite materiam», uno dei luoghi oraziani più glossati nel Medioevo e in particolare nella tradizione retorica, che Dante usa per incitare i lettori a ponderare il peso della materia alle proprie spalle per evitare di cadere («ununquenque debere materie pondus propriis humeris coequare, ne forte humerorum nimio gravatata virtute in cenum cespitare necesse sit» II, iv, 4). ${ }^{30}$ Più espliciti ancora i passaggi inseriti in quello che si è definito cripto-manuale di dictamen, e cioè il citato paragrafo sulla costructio, dove Dante prorompe due volte in invettive contro coloro che, prima di lui, hanno praticato la canzone senza consapevolezza: una volta sono definiti ydiote («pudeat, ergo, pudeat ydiotas», II, vi, 3, con termine molto forte e riferimento alla cecità di questi “idioti”, che salda il passaggio al prologo del trattato), ${ }^{31}$ quindi, dopo la più lunga esemplificazione presente nel trattato e consistente in ben 11 canzoni illustri in occitano, francese e italiano, ${ }^{32}$

Alessio ad locum che segnala il motivo del clericus che "privo di libri "ambulat in tenebris"», (Bene da Firenze, Candelabrum, p. 294).

29 Guido Faba, Summa, p. 288; cfr. anche la Gemma purpurea (1239-1248): «nam ecce philosophie palatium aperio clavibus michi datis, et misericordia ductus, vinculis resecatis, extraho de manibus impiorum materias nequiter laceratas, quas magni archimandrite ordiri nesciunt et variare ignorant per gradus, ordines et personas, ab eadem captivitate nichilominus liberans adverbia cum verbis et participia cum lucidis adiectivis, et ad studentium honorem, gaudium, profectum et gloriam disponens artificialiter singula et sedi proprie, dignitati, honori et loco restituens universa, in quibus omnis homo palpans clamabat nec erat qui adiuvaret, errabat Arrialdus et maximum defectum cetus scolasticus sustinebat». Cito da Vescovo, La Gemma purpurea di Guido Faba.

30 Come si è detto, la citazione oraziana risulta essere tra le più commentate nella tradizione medievale, solitamente a cerniera della trattazione precettiva nell'Ars poetica (Villa, Dante lettore di Orazio).

31 Il passaggio è raccordato anche agli «idioti che non saprebbero l'abc» di Conv. IV, xv, 16. 32 Per un'analisi sintattica di questo corpus esemplificativo - il più corposo del Dve - cfr. almeno Chersoni, Tavoni, Ipotesi d'interpretazione, pp. 142-157. 
sono chiamati "seguaci dell'ignoranza” e identificati negli imitatori di Guittone d'Arezzo («subsistant igitur ignorantie sectatores Guictonem Aretinum et quosdam alios extollentes», II, vi, 8). ${ }^{33}$

Non si è mai notato come anche queste invettive rientrino nella linea dei maestri bolognesi, e anzi addensano rimandi al già ricordato Guido Faba: nell'Ars di quest'ultimo, il «sumite materiam» oraziano è adibito alla modellizzazione del «dictator sagax (...) diligens et discretus ad inveniendam materiam suo ingenio congruentem»; $3^{34}$ le invettive specificamente anti-guittoniane contribuiscono di conseguenza a rinforzare l'autopresentazione di Dante come dictator illustre sul piano del volgare con un forte richiamo al fenomenale prologo autobiografico dello stesso testo, dove l'annuncio della propria opera è presentata come lo strumento che supererà gli antichi errori e permetterà all'uomo, edotto dalla grazia di questa sapienza miracolosa, di non essere corrotto dalla ignoranza e dalla cecità: «ecce novella surrexit gratia, abicite procul vetustatis errores, ut viri doctissimi sollicite precaventes ne ignorantie vel cecitatis fermento massa vestre prudentie corrumpatur». ${ }^{35}$

Collocarsi nella linea di Guido Faba serve a Dante per esplicitare la propria visione del dictamen rispetto ai due più importanti autori in volgare della generazione precedente alla propria, Brunetto Latini e Guittone d'Arezzo. Entrambi questi autori avevano fornito una propria interpretazione di questo sapere egemonico, nonché una sua particolare applicazione pratica, in due contesti (Firenze e Arezzo) in cui la rettorica aveva assunto un'importanza cruciale nella vita cittadina. Ciò che accomuna queste due esperienze è un progetto di totale volgarizzazione, attuato parallelamente ma diversamente.

Brunetto fa convogliare nel dictamen la letteratura pragmatica e morale e la tradizione concionatoria e si investe (più o meno) personalmente nella diffusione di un corpus di volgarizzamenti che affianca i grandi dictamina svevi ai discorsi dell'antichità (alcune orazioni ciceroniane), ma si impegna anche nel commento in volgare del De inventione (pure servendosi di tradizione glossatoria di scuola nella incompiuta Rettorica e nell'enciclopedia del Tresor). ${ }^{36}$ Questa trasformazione "dall'interno" della tradizione retorica influenzerà nella lunga durata l'ambiente culturale fiorentino, rendendo l'esperienza dantesca, come

33 E su questo punto, si veda almeno Antonelli, “Subsistant igitur ignorantiae sectatores".

34 Guido Faba, Summa, p. 334.

35 Guido Faba, Summa, p. 287. E si veda anche il Prologus della Rota nova, che riprende il topos del "superamento" in maniera originale: «taceat igitur totus mundus, et Tulliana peritia necnon et facundia Ciceronis loquatur, et a finibus terre austri regina veniat audire sapientiam Salomonis» (Magistri Guidonis Fabe Rota nova, p. 6).

36 Cfr. soprattutto Alessio, Un commento in volgare. 
spesso succede, isolata o comunque parallela e irriducibile a quella. ${ }^{37}$ Guittone d'Arezzo, pure avulso da ogni teorizzazione, realizza un vero e proprio (il primo, e a lungo l'unico) epistolario volgare d'autore, preservato dal codice Laurenziano Rediano 9 in uno con le canzoni morali secondo un'organizzazione voluta dal copista (che infatti rubrica «Lettere e cansone»). ${ }^{38}$ Oltre al dato linguistico, ciò che caratterizza l'epistolografia guittoniana è questo incerto confine tra poesia e prosa, di tipo sia macrostrutturale - alcune poesie sono inserite nella sezione delle lettere - sia microstrutturale - la prosa epistolare è caratterizzata da tratti ritmici che lo avvicinano alla scrittura in versi. ${ }^{39}$

Il distanziamento dantesco da queste due esperienze è forte ed esplicito. Per quanto l'interpretazione della condanna dei sectatores ignorantiae, soprattutto per l'incertezza che vige nell'accomunare la lunga lista di undici esempi di canzone illustre nella sezione della constructio, è ancora oggetto di discussione, non escluderei ${ }^{40}$ che l'invettiva contro i guittoniani cumuli, per Dante, anche questa eccentrica interpretazione del dictamen, sul piano sia linguistico sia dei generi letterari. Così, anche contro il Latini che aveva addebitato alla scrittura in versi una certa oscuritate ${ }^{41}$ che la rendeva inferiore alla prosa, Dante non manca di collocare l'intera sua trattazione all'ombra dell'idea della superiorità della poesia, promuovendo il tipo metricum come exemplar rispetto a quello prosaycum e dunque dotandolo di un sicuro primatus (Dve II, i, 1). La posizione è piuttosto eccentrica rispetto alla tradizione delle artes, dove la produzione in versi volgare rientra nel genere rithmicum (e il trattato ad essa dedicato da Antonio da Tempo nel 1332 ca. si intitola conseguentemente Summa artis rithmici vulgaris); conguagliando la poesia volgare sotto l'ombrello di quella quantitativa classica, ${ }^{42}$ l'Alighieri conferma, in maniera paradossale, la sua totale compartecipazione alla mentalità dei maestri bolognesi di dictamen finanche nel suo

$37 \mathrm{Su}$ questo tema, che ha suscitato qualche discussione, mi permetto di rinviare a Bischetti, Montefusco, Prime osservazioni, in part. alle pp. 182-193, dove si discutono anche le tesi di von Moos, che tendevano a vedere nel Latini una cultura completamente divaricata da quella di impianto "dictaminale": Von Moos, Die italienische ars arengandi.

38 Carrai, Guittone e le origini. Si veda anche Leonardi, Guittone e dintorni.

39 Lo ha studiato con sistematicità Pasquini, Intersezioni.

$40 \mathrm{Mi}$ rendo conto che dello statuto del tutto ipotetico della proposta; per l'interpretazione corrente, si veda Antonelli, "Subsistant igitur ignorantiae sectatores".

41 Nel Tesoretto Brunetto dice: «quando vorrò trattare / di cose che rimare / tenesse oscuritate / con bella brevitate / ti parlerò per prosa» (si cita da Brunetto, Poesie, vv. 419-423).

42 In Dve II, i, 1; II, vii, 7; II, xi, 4 Dante usa "metricus" per la versificazione in volgare, riservando "rithimus" per la rima, con uso diverso dalla manualistica (per esempio, lo stesso Antonio da Tempo) che intendeva con quest'ultimo termine la versificazione volgare: $\mathrm{cfr}$. il commento di Tavoni in Dve (Tavoni), II, i, 1. 
progetto di nobilitazione del volgare illustre e, di riflesso, spiega almeno in parte il motivo di quella pagina del trattato che modellizza la constructio excelsa su modelli prosastici. L'esempio più calzante, per una tale forma mentis, è di nuovo Guido Faba, che al culmine della carriera inizia a fornire, all'interno dei suoi manuali, modelli di lettere in volgare (nella Gemma purpurea e nei Parlamenta et epistole), aprendo alla fortunata summa interamente in volgare composta, sempre nell'ambiente bolognese, da Matteo de' Libri. ${ }^{43}$

Di fronte, dunque, alla pressione dell'ascesa e dell'affermazione del volgare, i maestri sviluppano un progetto che pretende di "governare" queste nuove tendenze culturali, tenendo ben salda la gerarchia linguistica in ragione di un'idea di dictamen come linguaggio artificiale, che si avvicina a quello divino e quindi necessariamente latino. ${ }^{44}$ Se Brunetto e Guittone avevano fatto saltare questa gerarchia, Dante la erode dall'interno, restando fedele a una epistolografia in latino redatta secondo uno stile personale ma comunque precisamente inquadrabile nelle tendenze del tempo, e allo stesso utilizzando questa competenza per affermare e dimostrare geometrico more la possibilità cognitiva di una poesia volgare illustre.

\section{Autocoscienza teorica e prassi epistolare}

Inquadrato, dunque, il know how retorico-dictaminale che possiamo definire specificamente dantesco, è ora importante verificare se l'Alighieri si mantenga conseguente nella prassi scrittoria, e cioè nelle sue lettere, e additivamente collocare la sua di fronte a esempi coevi. Si tratta di un tentativo non ancora realizzato; proverò, dunque, a proiettare la modellizzazione del Dve sul testo epistolare, cercando di mostrare come tale teorizzazione e la pratica epistolare, pur non concretizzandosi in automatica "traduzione tecnica" del modello mentale che Dante ha in testa, risultano comunque coerenti e appartenenti a un timbro d'autore; in particolare, mi preme verificare come e quanto le constructiones con le quali sono costruite le epistole siano conseguenti rispetto agli esempi prosastici di Dve II, vi, 4-5.

Nell'impossibilità di analizzare l'intero corpus, mi limito a mostrare un passaggio esemplificativo. Lo estraggo da un pezzo particolare ma molto rappresentativo, e cioè l'Ep. V, una circolare rivolta ai poteri e alle istituzioni italiane

43 Innovazione tanto più significativa, se a Matteo sono attribuite anche summae integralmente in latino: Kristeller, Matteo de' Libri.

44 Grévin, L’ars dictaminis, discipline hégémonique, pp. 32-33. 
(ordinatamente: i re, in quel momento di Napoli e di Sicilia; i senatori di Roma; i funzionari e le comunità del Regnum Italicum). Il tema della lettera è l'annuncio della futura missione con cui Enrico di Lussemburgo, eletto re dei Romani nel 1308, si dirige a Roma per indossare la corona imperiale e ricevere dal papa unzione e consacrazione: il pontefice Clemente $\mathrm{V}$ ne annuncia l'incoronazione in San Pietro con una bolla del 1310. Come si comprende subito, il tema e i mittenti esigono uno stile tragico, intonato al dictamen illustre, per il quale si richiamano spesso i grandi manifesti della cancelleria sveva, redatti sotto il controllo di Pier della Vigna. ${ }^{45}$ Leggiamo ora l'exordium della lettera, la parte, cioè, che precede la narratio, considerata il nucleo del testo, di cui si sono numerati, per la comodità dell'analisi, i periodi.

«Ecce nunc tempus acceptabile», quo signa surgunt consolatiònis et pàcis [pl]. (1) Nam dies nòva splendèscit $[p l]$ ab ortu auròram demònstrans $[p l]$, que iam tènebras diutùrne $[v l]$ calamitàtis attènuat $[v l]$; (2) iamque aure orientàles crebréscunt $[p l]$, rutilat celum in làbiis sùis $[p l]$, et auspitia gentium blanda serenitàte confòrtat $[p l]$. (3) Et nos gaudium expectàtum vidébimus [td], qui diu pernoctitàvimus in desérto [vl], quoniam Titan exoriétur pacìficus $[t d]$, // et iustitia, sine sole quasi eliotròpium hebetàta, cum primum iubar ille vibràverit, revirèscet $[v l]$. (4) Saturabuntur omnes qui esuriunt et sitiunt iustitiam in lumine radiorum eius, et confundentur qui diligunt iniquitatem a fàcie coruscàntis $[v l]$. (5) Arrexit namque aures misericordes Leo fòrtis de tribu lùda [vl]; // atque ullulatum universalis captivitatis miserans, Moysen àlium suscitàvit [vl] qui de gravaminibus Egiptiorum populum sùum erìpiet $[t d]$, ad terram lacte ac melle manàntem perdùcens $[\mathrm{pl}]$.

Lascio da parte il primo periodo, che contiene una citazione illustre, chiamata sententia o proverbium (in questo caso, Dante richiama una lettera paolina), una delle modalità consigliate per dare avvio a una epistola (nelle lettere dantesche, l'indicazione è rispettata con costanza). I cinque periodi successivi si possono agevolmente comparare con le constructiones modello di Dve II, vi, 4-5 per lunghezza, arcatura retorica e tessitura ritmica. Essi infatti contengono due membri di estensione comparabile $(1,2,4)$ talvolta raddoppiati o leggermente allungati (3 e 5: li si è indicati con //); essi non sono rigidamente isosillabici, come nella constructio sapida et venusta, ma leggermente variati, come nella excelsa. Vagliamo in prima battuta la costruzione delle clausole ritmiche.

45 L'edizione del testo è ora in L'epistolario di Pier della Vigna; l'influsso della scrittura emanante dalla cancelleria sveva nella storia del dictamen è studiato in Delle Donne, Una costellazione; Delle Donne, La cultura; Grévin, Rhétorique du pouvoir; Delle Donne, Le lettere. Sulla diffusione in Toscana, cfr. B. Grévin, Héritages culturels des Hohenstaufen. L'influsso delle lettere sveve (in particolare la Collegerunt pontifices, che si legge in L'epistolario di Pier della Vigna, I, 1, pp. 79-92) sulle lettere arrighiane è sistematizzato in Grévin, Rhétorique du pouvoir, pp. 795-802. Una nuova fonte per la lettera XI è ora oggetto di un intervento di Delle Donne, Una fonte. 
L'ornamentazione applicata alle clausole, interne e finali, della prosa offriva al dictator esperto uno strumento di scansione sintattica nonché di innalzamento stilistico, basato su un repertorio a cui poteva attingere tramite la mnemotecnica, e che consisteva in una serie di sintagmi (perlopiù composti da due parole ma talvolta, tramite consillabicatio, comprendente anche bisillabi o monosillabi, che andavano a scomporre l'unità ritmica costituita dalla parola) che potevano essere variati attingendo, per esempio, alle desinenze della flessione nominale o verbale, oppure usando parole con radici assimilabili, stavolta desunte perlopiù dalle summae. ${ }^{46}$ Tenendo presente questa risorsa, andranno presi come termine di paragone i due esempi di constructiones corrette e sviluppate con un minimo («superficietenus») di retorica in Dve II, vi, 4-5, e cioè l'esempio eccellente, che presenta due tardus chiusi da un velox: «eiecta maxima parte florum de sinu tùo Florèntia $[t d]$, nequìcquam Trinàcriam $[t d]$ Totila secùndus adivit [pl]»; e, prima, l'esempio «sapidus et venustus» che incornicia un velox all'interno di due planus: «laudabilis discretio marchiònis Estènsis [ $p l]$ et sua magnificéntia préparata $[v l]$ cunctis, cunctis illum facit èsse diléctum $[p l] »$.

Nel testo citato di Ep. V, 1-5 mi pare significativo rilevare come le constructiones siano scandite in linea di massima su tre pause dotate di cursus; nel caso del periodo 3 si sottolinea la presenza di uno schema in cui le tre clausole si dispongono in simmetria, chiudendo il velox all'interno di due tardus; prediletto sembra le schema che tende a variare le clausole (in 1 e 5), con l'ovvia conseguenza che, laddove il periodo tende ad allargarsi, si creano voluti effetti di simmetria (negli stessi casi 1 e 5). La ripetizione su planus (in 2) è evidentemente a parte, distillata da una ricerca di accumulatio che tende al parallelismo tra i vari elementi naturali che partecipano all'avvento di Enrico. Per quanto, dunque, non si possa parlare di sovrapposizione, la modellizzazione e la pratica epistolare di Dante sono in stretto rapporto tra di loro sul piano della ricerca di scansioni ritmiche della frase, con una certa attenzione alla simmetria.

Un parallelo discorso può farsi sul piano dell'ordo. Nel testo epistolare, si noti almeno la differente dislocazione del verbo prima in posizione naturale poi finale in 1; in 2 finale e, immediatamente contiguo, iniziale e finale e così via, in una variatio che non induce all'obscuritas. Delle figure retoriche, si rilevino la continuata transumptio dell'aurora, in disiunctio con le immagini di tenebra, nonché le antonomasie per l'imperatore (Titan e Moyses). ${ }^{47}$ Qui si può verificare una forte tendenza al modulo della constructio excelsa, che «si differenzia dai

46 Cfr. Grévin, L'empire d'une forme, e Grévin, Le Epistole dantesche e la prassi duecentesca dell'ars dictaminis, in questo volume.

47 Per il ruolo di questa figura, detta pronominatio, si veda il saggio di Tomazzoli in questo volume. 
precedenti soprattutto per l'uso della tecnica metaforico-simbolica continuata della transumptio, culmine dell'ornatus difficilis». ${ }^{48} \mathrm{Ma}$, di nuovo, si attinge anche al periodare sapidus et venustus «proprio di coloro che amano servirsi dei "colores rhetorici"». ${ }^{49}$

Resta da verificare se tale uso, con l'addentellato della sua autocoscienza teorica, produca oppure no un timbro originale nel contesto retorico-epistolografico del tempo. Tenendo presente una certa oscillazione dovuta ai problemi di un testo critico non ancora affidabile né stabile, ${ }^{50}$ nel corpus superstite emerge con una certa evidenza una concorrenza tra la clausola ritmica raccomandata per le pause forti, e cioè il velox (pp4p), e il tardus (p4pp). Già nei sintagmi in posizione finale Dante tende a proporre il tardus circa una volta su cinque, e questo uso tende a salire considerando i periodi interni fino a uno su tre, quasi pareggiando il velox (parliamo di circa 445 clausole con tardus, laddove si riscontrano, per il velox, una ventina di esempî in più). ${ }^{51}$ Nella storia del cursus alcuni casi isolati presentano una spiccata preferenza per il tardus: si possono citare il papa riformatore Gregorio VII (†1085) nonché, nella manualistica, le indicazioni definite nei Praecepta dictaminum di Adalberto Samaritano (11111118), uno dei primi maestri bolognesi; ${ }^{52}$ siamo in una fase (secc. XI-XII in.) cronologicamente piuttosto alta della storia dell'epistolografia medievale, e sicuramente travolta dall'uso sempre più dominante del velox che, tra XIII e XIV secolo, tenderà a ricacciare il tardus a un'occorrenza più ridotta. ${ }^{53}$

L'Alighieri, tuttavia, non presenta un così forte rovesciamento delle gerarchie ritmiche, che ha indotto qualche studioso - tra cui l'illustre Tore Janson ${ }^{54}$ -

48 Dve (Mengaldo), II, vi, 4, commento ad locum.

49 Dve (Marigo), II, vi, 4, commento ad locum.

50 Sui problemi filologici delle edizioni delle lettere di Dante, mi permetto di rimandare alla ricostruzione in Montefusco, Le "Epistole”, pp. 412-416; ugualmente, per una valutazione delle edizioni più recenti (Pastore Stocchi, Villa, Baglio), rimando a un intervento-recensione: Montefusco, Epistole a c. di M. Baglio. L'uso del cursus a scopo ecdotico nella restitutio delle epistole dantesche, e non solo, è discusso in Chiesa, L'impiego del cursus, pp. 293-301 (con importanti osservazioni anche sull'uso dantesco).

51 Il calcolo è realizzato a partire da Rossetto, L'uso del "cursus", in particolare nelle tabelle riassuntive di pp. 63-71; questo esame, l'unico finora complessivo, abbisogna forse di qualche aggiustamento (in particolare nella tendenza a valorizzare intrecci e catene, e talvolta a individuare clausole ritmiche in pause minori del periodo), ma è ancora uno strumento di base di certa utilità.

52 Per l'uso della cancelleria papale al tempo di Gregorio VII, Janson, Prose, pp. 42-49; per la preferenza accordata al tardus da Adalberto Samaritano, eccezionale nella storia del dictamen, si veda p. 77.

53 Janson, Prose Rhythm, pp. 69-ss.

54 Janson, Prose Rhythm, pp. 79-80. 
a ipotizzare una specifica tradizione scolastica fiorentina distinta, su questo piano, dalla tradizione bolognese nonché da quella affermatasi oltralpe. In Dante si riscontra invece un equilibrio fra i tre tipi di clausole (incluso, dunque, anche il planus, p3p, usato ca. il 30\% delle volte in chiusura di periodo) con una spiccata ricerca di parallelismi nella tessitura del periodo che, come abbiamo appena visto, trova specifica esplicitazione nel capitolo della constructio del Dve. Si prenda la lettera solenne scritta da Dante all'imperatore Enrico VII il 17 aprile 1311, in un momento di forte stallo della campagna militare dovuto alla ribellione delle città lombarde. A conclusione della petitio, dopo aver individuato nell'azione di Firenze un tentativo di dilazione che vorrebbe sfiancare l'operazione imperiale, l'Alighieri indica nel figlio di Enrico, Giovanni di Boemia, un nuovo Ascanio, quindi una sorta di erede "provvidenziale" del ruolo del padre, la cui carica imperiale, è bene ricordarlo, era elettiva. Il passaggio è dunque cruciale per il discorso dantesco, che ritaglia all'impero un posto cruciale nel progetto di Dio sulla terra.

Iohannes namque, regius primogenitus tuus et rex, quem, post diei orièntis occàsum $[p l]$, mundi successiva postèritas prestolàtur $[v l]$, nobis est àlter Ascànius $[p l]$, // qui vestigia magni genitòris obsèrvans $[p l]$, in Turnos ubique sicut lèo desèviet $[t d]$ et in Latinos velut àgnus mitèscet $[p l] . »(E p$. VII, 18).

In questo passaggio elegantemente accordato si noterà come le due constructiones si poggiano su due clausole con cursus planus che incorniciano una volta un velox e un'altra un tardus; si tratta di una struttura ritmica intesa a rilevare parallelismi o disgiunzioni intense (come quella tra i seguaci di Turno e gli epigoni dei Latini, intese a sottolineare il doppio approccio richiesto al governo imperiale, di dura reazione a chi si ribella e di mitezza nei confronti di chi è fedele) dotati sempre di forte significato. Non si vuol dire qui che sia impossibile trovare qualche esempio di prosa epistolare che presenti un similare equilibrio tra le clausole. ${ }^{55}$ Ma ciò che sembra proprio di Dante è un sistematico ricorso a strutture ritmiche più complesse, costruite volontariamente su ripetizioni e contrasti e che si dispiegano sull'intero periodo, con uno scopo non solo sintattico ma più sensibilmente semantico.

55 Può qui essere ricordato il caso del papa Alessandro II (†1073), predecessore di Gregorio VII, in una fase di assestamento dello stile della cancelleria papale: Janson, Prose Rhythm, pp. 47-49. 


\section{Uno stile curiale}

A partire da questo dato, possiamo ora provare a precisare la posizione della prassi dictaminale rappresentata dalle lettere dantesche a fronte di quelle coeve. La letteratura critica ha, su questo punto, avanzato proposte diverse e talora non totalmente coerenti o contraddittorie tra di loro. Spesso si è avuta la tendenza ad avvicinare lo stile dantesco a quello supremus usato nella cancelleria imperiale, e in particolare nello stile delle lettere più o meno riconducibili all'autorità di Pier della Vigna. La conoscenza di questi testi da parte di Dante, oltre che attesa, è senza meno facilmente dimostrabile a partire dal canto XIII dell'Inferno, dove il pellegrino incontra l'anima del logoteta imperiale. In bocca a Piero viene messo un discorso in versi (volgari) che riprende puntualmente le caratteristiche più salienti del dictamen svevo: sul piano dell'ornatus difficilis, risalta l'uso di transumptiones caratteristiche di questi testi, quali quella della vigna (utilizzato nell'elogio del logoteta realizzato da Nicola della Rocca), quella della meretrice e quella della chiave, accanto ai poliptoti su "infiammare" e “credere", le figure di suono, l'iniziale anafora dispiegata su tre terzine e rime ricercatissime al limite dell'omofonia. ${ }^{56}$ Se pensiamo che il discorso di Piero rispetta la partizione epistolografica classica (exordium ai vv. 55-57; narratio ai vv. 58-75), con la petitio (vv. 76-78) consistente nella richiesta di confortare la memoria del dannato sulla terra, ne esce confermata l'idea che quello di Dante è, innanzitutto, un discorso critico sulla specifica prassi retorica della Magna Curia: si intende che, per l'Alighieri, questo stile sovraccarico, che pone la prosa latina in concorrenza diretta con la poesia in ragione dell'accumulo di colores retorici e usi ritmici, è un aspetto "negativo" della scrittura della cancelleria sveva e dei suoi principali esponenti. ${ }^{57}$ In questo senso il giudizio dantesco sembra convergere con alcuni contemporanei che avevano sottolineato la conseguenza negativa, sul terreno della comprensione, di questa prosa di natura propagandista e politica, parlando di obscuritas. ${ }^{58}$ Non sorprende, dunque, di scoprire che lo stile epistolare di Dante non sembra avvicinabile a quello del logoteta; e nemmeno i riscontri puntuali tra le due opere sembrano andare al di là di un ristretto gruppo di stringhe testuali che insistono su fonti comuni. ${ }^{59}$

56 Villa, «Per le nove radici d'esto legno».

57 Jacomuzzi, Il palinsesto della retorica, parla genericamente di una critica della retorica in generale, anzi di una sua «dissacrazione» (p. 66).

58 Per il giurista Odofredo, lo stile di Piero è caratterizzato da un «obscure loqui» (Kantorowicz, Über die dem Petrus, p. 653 nota 1).

59 Cfr. su questo tema la nota 46. 
Sembra anche fuorviante e anacronistico comparare lo stile di Dante con quel gruppo di stili che sembrano perlopiù legati a contesti di insegnamento della retorica, definiti "romano" (che privilegiava un uso localizzato del cursus) o "ilariano" (che invece generalizzava il ricorso al ritmo), e poi "ciceroniano" (che attinge specialmente alle risorse dell'ornatus) e "isidoriano" (caratterizzato dall'uso di figure di suono). Nella tradizione delle poetriae, e in particolare nella Parisiana poetria di Giovanni di Garlandia (1220-1235), questi quattro stili «modernorum» indicano le differenti possibilità di arricchimento della prosa latina. ${ }^{60}$ Bisogna però ammettere che nella scrittura di Dante risulta davvero difficile individuare la prevalenza di uno di questi stili, sebbene sia utile rimarcare, come ha ben esemplificato l'ultimo commentatore Marco Baglio, che il cosiddetto isidoriano «è lo stile che Dante mostra di preferire, incrociandolo con la prosa ritmica tipica dello stile romano. Ripetizioni, poliptoti e paronomasie segnano in generale la prosa latina di Dante e nello specifico quella epistolare». ${ }^{61} \mathrm{Ma}$ l'abbondantissimo ricorso all'ornatus, specie difficilis, orienta anche verso lo stile che si attribuisce a Cicerone, sebbene proprio quest'ultimo costituisca in qualche modo la prova della sfocatezza di tale paradigma, dato che con stilus tullianus si indicava la seriazione dei colores desunte, nell'ars dictaminis, dalla pseudo-ciceroniana Rhetorica ad Herennium. ${ }^{62}$

Il quadro di pratiche stilistiche che sembra giustificare con più precisione la scrittura epistolare dantesca è da individuare nel cosiddetto stylus Curie romane, che da qualche studioso illustre è stato definito, con ulteriore anacronismo, stylus rhetoricus. ${ }^{63} \mathrm{Si}$ tratta dello stile cancelleresco che si è sviluppato e affermato presso la cancelleria papale durante un lungo percorso evolutivo, giunto a maturazione durante il XIII secolo, perfezionandosi durante il pontificato di Onorio III e poco dopo. Esso è il frutto del trapianto in ambito papale

60 Sembrerebbe trattarsi di una "invenzione" di Giovanni di Garlandia, il quale pone sotto queste etichette i quattro possibili stili di cancelleria: «de quatuor stilis curialibus. Preter stilos tres poeticos et de pedibus servandis in dictamine. Item preter tres stilos poeticos sunt et alii stili quatuor quibus utuntur moderni, scilicet Gregorianus, Tullianus, Hyllarianus, Hysydorianum» (The Parisiana Poetria, c. 5, 1. 402). Lo ha mostrato Turcan-Verkerk, La théorie des quatre styles, che ha anche sottolineato il corto-circuito che si è ingenerato nella tradizione di studi moderna, che ha dato largo credito a questa costruzione: si veda, ad esempio, l'articolo di Schiaffini, Gli stili prosastici e Plezia, Quattuor stili modernorum.

61 Ep. (Baglio), p. 22.

62 Destinato a grande fortuna, come si sa, ma in epoca più tarda. Per Giovanni di Garlandia: «in stilo tulliano non est observanda pedum cadencia, set dictionum et sententiarum coloracio. Quo stilo utuntur vates prosayce scribentes et magistri in scolasticis dictaminibus» (The Parisiana Poetria, c. 5, 1. 432-433).

63 Witt, Medieval “Ars dictaminis”, p. 30; la categoria è stata criticata da Grévin, Rhetorique du pouvoir, p. 135 n. 46. 
e della conseguente trasformazione dello stile teorizzato nella scuola francese (detta “di Orléans”), caratterizzato dall'impiego di ricchissime serie di figure retoriche e da un risultato prosastico piuttosto analitico; a questi influssi si affiancarono anche gli apporti provenienti dalla scuola bolognese, nonché dalla tradizione meridionale. Lo stile della curia papale trovò la sua individualità nel forte ricorso a meccanismi di pathos, desunti dalla tradizione omiletica e specificamente cistercense (legata alla predicazione per le crociate) - caratteristica particolarmente adatta alla performance orale di lettura collettiva e pubblica in cerimonie solenni. ${ }^{64}$

Prendiamo un esempio particolarmente rappresentativo: una delle lettere raccolte nella Summa dictaminis di Tommaso da Capua. Durante i pontificati di Innocenzo III, Onorio III e Gregorio IX, Tommaso ebbe un ruolo centrale nella cancelleria papale, contribuendo a definire lo stylus curie romane e influenzando a sua volta l'epistolografia sveva tramite il complesso di esperimenti stilistici latini realizzati da un nutrito gruppo di dictatores campani, e più precisamente capuani, all'inizio del Duecento. ${ }^{65}$ Ecco un passaggio di una epistola che ha per destinatario un religioso e che ruota intorno al concistoro:

O felix, quem perplexa temporalium retia non involvunt! O felix, quem fluvius desiderii terrenorum immissum a bestia retardare non potuit, qui liberis pennis et siccis pedibus ab humore carnalium voluptatum exemptus volaveris in solitudinem, qua quiescis! Ecce, nunc sedes ad pedes Domini cum Maria; ecce, nunc in Rachelis pulchritudine delectaris; ecce, iam gustas, quam magna multitudo dulcedinis, quam abscondit Deus diligentibus se! Quid plura? Manifeste loquor, publice fateor et aperte protestor, quod factus es ante me sinceritate conscientie, innocentia vite, fame decore. ${ }^{66}$

Sul piano dell'ornatus difficilis, si noterà la transumptio delle preoccupazioni temporali rappresentate come delle reti, a cui si lega l'immagine del volo di chi se ne libera; così, la metafora del fiume, anch'essa a rappresentazione delle conseguenze negative del secolo, resta isolata ma ben evidenziata nel suo contrasto con la rappresentazione aerea precedente. Il ritmo è costruito tramite una serie di ripetizioni ( $O$ felix ... ecce, nunc ... quam ...), interrogative retoriche (quid plura?) ed esclamative, con una climax di impianto patetico che ha uno scopo evidentemente oratorio. Rientrano in questa complessiva intenzione anche le figure di suono qui sfruttate, tra le quali emergono soprattutto gli esibiti omoteleuti e assonanze (i più evidenti sono loquor ... fateor ... protestor, ma non va

64 Schaller, Studien zur Briefsammlung, pp. 371-51; cfr. anche l'introduzione di Delle Donne in Nicola da Rocca, Epistole, pp. XXIII-XXVI.

65 Delle Donne, Tommaso di Capua.

66 Die Briefsammlung des Thomas von Capua, p. 55. 
sottovalutato anche l'accumulo di suoni sibilanti nell'immagine del volo: liberis pennis et siccis pedibus ... volaveris ... quiescis).

Per verificare l'operatività di tale modello, facciamo riferimento a un pezzo, tra le epistole dantesche, che risulti omogeneo per il tema ma anche per il carattere parenetico, e cioè l'epistola XI. La lettera è redatta da Dante durante il drammatico conclave di Carpentras, interrotto manu militari da un'irruzione organizzata dal gruppo di cardinali guasconi per evitare che il papa ritorni a Roma. L'Alighieri, che pure è privo di qualsivoglia legittimità pastorale («nulla pastorali auctoritate abutens», Ep. XI, 9), osa prendere la parola divorato dallo zelo e confortato dagli esempi di coloro che, nonostante fossero umili o addirittura lattanti, hanno saputo vedere la verità e mostrarla ai farisei, renitenti a vederla. L'intenzione è contraria a quella dei guasconi: incitare i cardinali italiani a combattere («viriliter propugnare», 26) per riconquistare alla città di Roma e all'Italia la sede petrina. Nel cuore della narratio, dopo aver spiegato ai propri interlocutori che egli scrive, pure minima pecora del signore, perché dotato della grazia, Dante prorompe in un'invettiva che descrive una curia in preda alla cupidigia e l'iniquità, in cui le opere dei padri e dei dottori sono abbandonati a favore della ricchezza e dei benefici:

Quidni? Cupiditatem unusquisque sibi duxit in uxorem, quemadmodum et vos, que nunquam pietatis et equitatis, ut caritas, sed semper impietatis et iniquitatis est genitrix. A, mater piissima, sponsa Christi, que in aqua et Spiritu generas tibi filios ad ruborem! Non caritas, non Astrea, sed filie sanguisuge facte sunt tibi nurus; que quales pariant tibi fetus, preter Lunensem pontificem omnes alii contestantur. Iacet Gregorius tuus in telis aranearum; iacet Ambrosius in neglectis clericorum latibulis; iacet Augustinus abiectus, Dionysius, Damascenus et Beda; et nescio quod "Speculum”, Innocentium, et Ostiensem declamant. Cur non? Illi Deum querebant, ut finem et optimum; isti census et beneficia consecuntur (Ep. XI, 14).

Il testo è costruito intorno alla corposa transumptio del matrimonio tra i cardinali e la cupiditas, a partire dal quale si generano le immagini della cupidigiamadre e delle figlie della sanguisuga-nuore. Per intensificare il pathos, Dante puntella la lettera di interrogative retoriche (quidni? ... cur non?), intrecciandole con una ricca serie di esclamative. Un medesimo intento di costruzione di un ritmo quasi martellante è da indicare nell'uso abbondante di omotoleuti nella prima parte del passaggio (pietatis ... equitatis ... impietatis ... iniquitatis), interrotti da due termini con sibilante finale (caritas ... genitrix). Nella seconda parte, è l'anafora (iacet ... iacet) a sfociare in due liste (Dionysius, Damascenus et Beda ... "Speculum”, Innocentium, et Ostiensem) e nella contentio finale che contrappone Padri e Dottori agli attuali cardinali. 


\section{La legittimità del dictator}

Gli elementi che abbiamo raccolto sono utili per precisare l'attività del Dante dictator, per individuare i punti di maggiore originalità della sua produzione epistolare, per individuare quale ruolo ebbe il dictamen come sapere egemonico e pratico insieme nella sua opera. Dobbiamo sottolineare in prima istanza come emerga, dall'indagine condotta finora, una notevole coerenza tra l'autocoscienza dell'Alighieri esperto di epistolografia che abbiamo visto all'azione nel Dve e la prassi scrittoria sviluppata nelle lettere. Non bisogna infatti porre una barriera o una divaricazione tra lo stile curie romane e la manualistica dei grandi maestri bolognesi a cui Dante si attiene, seppure secondo una linea di innovazione “dall’interno”. Proprio il caso di Tommaso da Capua è, a questo riguardo, particolarmente significativo. Tommaso, infatti, insegnò nelle scuole dell'Italia settentrionale - è attestato un suo insegnamento a Vicenza - e la sua riflessione sulla scrittura epistolare fu un punto di riferimento per la grande manualistica bolognese. ${ }^{67}$ Nel Candelabrum di Bene da Firenze, è Tommaso difatti a fornire gli elementi di riflessione e di nomenclatura fondamentali per la partizione della lettera (nell'abbreviatio del libro V), ma anche per gli usi aggettivali e verbali a cui attenersi nelle varie parti (per esempio nel libro III a proposito della salutatio). ${ }^{68}$

Formatosi nella Firenze dominata dalla figura di Brunetto Latini, Dante si sottrasse volutamente alla trasformazione, volgarizzazione e moralizzazione del dictamen che proprio il Latini aveva consegnato alla incompiuta Rettorica e al Tresor. Egli invece rimase fortemente coerente a un quadro scolastico, che concepiva la scrittura epistolare gerarchizzata sul latino come lingua dell'autentificazione documentaria, e allo stesso tempo attingeva, trasformandole, a tradizioni di scrittura curiale recepite sempre in ambito bolognese. Una delle motivazioni di questa visione può proprio essere ricercata e trovata nella peculiare posizione sociale dell'Alighieri, che, a differenza di Brunetto ma anche di autori a lui contemporanei (da Pier della Vigna a Francesco da Barberino), non ha un cursus di studi regolare compiuto né alcun titolo di incardinamento istituzionale (non è, per esempio, un notaio come Brunetto o Francesco da Barberino). Dante è un dictator illustre privo di una fonte di legittimazione istituzionale al di fuori della sua scrittura volgare; non è un caso se egli, dunque, cercherà di dimostrare a un pubblico di lettori probabilmente accademici la possibilità di scrivere in un volgare illustre superiore al latino attingendo anche alla tradizione del dictamen sulla base di una conoscenza di prima mano di summae e artes.

67 Heller, Die Ars dictandi.

68 Bene da Firenze, Candelabrum, pp. 339. 
Nella prassi dantesca il dictamen resta, di conseguenza, fortemente ancorato a un quadro di emissione istituzionale, che talvolta arriva fino ad obliterare la firma d'autore: succede nella lettera redatta in nome dell'Universitas Alborum (Ep. I) o nel gruppo di testi epistolari scritte per la moglie di Enrico VII a nome di Gherardesca, la moglie di Guido Guidi, conte di Battifolle (Epp. VIII-X). ${ }^{69}$ Questo fenomeno, che non cancella mai il timbro unico dello scrittore, è però piuttosto sorprendente se lo si confronta con il paziente e caratteristico progetto di Dante di presentarsi come auctor. ${ }^{70}$ A parte la consolatoria redatta in morte di Alessandro da Romena ( $E p$. II) e le lettere accompagnatorie di componimenti poetici (Epp. III-IV), dove emerge l'io dello scrittore secondo la normale routine della scrittura epistolare privata, nelle grandi lettere circolari (quelle arrighiane e quella ai cardinali), redatte in un momento in cui la Commedia iniziava a circolare e a essere conosciuta, ${ }^{71}$ allo stile curiale, dunque universale, Dante aggiungerà anche una precisa auto-presentazione profetica. ${ }^{72}$ Nella lettera scritta ai Fiorentini intrinseci il 31 marzo per ribadire il carattere sacrale della missione di Enrico e accusare la città di Firenze di costituire un empio nucleo di resistenza a tale missione, Dante si auto-descrive con queste parole:

et si presaga mens mea non fallitur, sic signis veridicis sicut inexpugnabilibus argumentis instructa prenuntians, urbem diutino merore confectam in manus alienorum tradi finaliter, plurima vestri parte seu nece seu captivitate deperdita, perpessuri exilium pauci cum fletu cernetis (Ep. VI, 17).

Come ha chiarito Elisa Brilli, l'espressione «praesaga mens» intarsia richiami virgiliani (Aen. X, 843) e agostiniani; pertiene anzi più precisamente ad Agostino la «definizione tecnica della mente profeticamente ispirata». ${ }^{73}$ In effetti, Dante qui lega la profezia all'interpretazione intellettuale di segni veridici, collocandosi quindi nel quadro definito del De Genesi ad litteram, dove, a partire dal commento al raptus Pauli e alla visione dell'Apostolo, Agostino esplicita una gerarchia tra i tipi di profezia secondo la quale è «maxime propheta» colui che è capace di attingere alla «vivacitate mentis» con la quale si interpretano le «rerum significativas similitudines». ${ }^{74}$ Questa gerarchia si incardina nella dot-

69 Cfr. su questo l'articolo di Attilio Bartoli Langeli in questo volume.

70 Ascoli, Dante and the Making.

71 Per le datazioni, si veda Inglese, Vita di Dante, pp. 118-ss.

72 Ledda, Modelli biblici. Per la convergenza di dictamen e profetismo nell'epistolario dantesco, cfr. Brilli, The interplay, pp. 153-69.

73 Montefusco, Le “Epistole”; sull'intertestualità Brilli, Reminiscenze scritturali, p. 550.

74 Agostino, De Genesi ad litteram, XII, ix, 20: «proinde, quibus signa per aliquas rerum corporalium similitudines demonstrabantur in spiritu, nisi accesserat mentis officium, ut etiam intellegerentur, nondum erat prophetia; magisque propheta erat, qui interpretabatur quod alius 
trina agostiniana dei tre tipi di visione, che posiziona l'intelletto (la mens), che vede oggetti incorporei, ${ }^{75}$ al di sopra dello spiritus e del corpo; questo quadro gnoseologico, che fu alla base della riflessione sulla profezia in ambito scolastico nel XIII secolo, ${ }^{76}$ approda all'idea per cui la visione intellettuale, che consta di un atto interpretativo, non sbaglia mai: «at vero in illis intellectualibus visis non fallitur: aut enim intellegit, et verum est; aut si verum non est, non intellegit: unde aliud est in his errare quae videt, aliud ideo errare quia non videt». ${ }^{77}$ Credo che si possa proporre l'ipotesi che questo specifico ragionamento sia alla base dell'idea dantesca di una «presaga mens (...) signis veridicis sicut inexpugnabilibus argumentis instructa prenuntians». La plausibilità di questo rimando, che mi pare non sia stato ancora rilevato, può essere confortata dalla tessera «non fallitur» che accompagna l'idea di una mente “presaga” (Ep. VI, 17).

Nella già citata lettera ai cardinali, Dante sente invece il bisogno di distinguere con forza la sua denuncia da quella di astronomi e profeti rozzi:

Piget, heu!, non minus quam plagam lamentabilem cernere heresium, quod impietatis fautores, Iudei, Saraceni et gentes, sabbata nostra rident, et, ut fertur, conclamant: «Ubi est Deus eorum?»; et quod forsan suis insidiis apostate potentes contra defensantes Angelos hoc adscribunt; et, quod horribilius est, quod astronomi quidam et crude prophetantes necessarium asserunt quod, male usi libertate arbitrii, eligere maluistis (Ep. XI, 4).

Il passaggio è di una certa importanza perché Dante accomuna $\mathrm{i}$ «crude prophetantes» a eretici e apostati, i primi soddisfatti dell'assenza di Dio che si verifica

vidisset, quam ipse qui vidisset. Unde apparet magis ad mentem pertinere prophetiam, quam ad istum spiritum, qui modo quodam proprio vocatur spiritus, vis animae quaedam mente inferior, ubi corporalium rerum similitudines exprimuntur. Itaque magis Ioseph propheta, qui intellexit quid significarent septem spicae et septem boves, quam Pharao qui eas vidit in somnis. Illius enim spiritus informatus est, ut videret; huius mens illuminata, ut intellegeret. Ac per hoc in illo erat lingua, in isto prophetia; quia in illo rerum imaginatio, in isto imaginationum interpretatio. Minus ergo propheta, qui rerum quae significantur, sola ipsa signa in spiritu per rerum corporalium imagines videt; et magis propheta, qui solo earum intellectu praeditus est: sed et maxime propheta, qui utroque praecellit, ut et videat in spiritu corporalium rerum significativas similitudines, et eas vivacitate mentis intellegat, sicut Danielis excellentia tentata est et probata, qui regi et somnium quod viderat dixit, et quid significaret aperuit. Et ipsae quippe imagines corporales in spiritu eius expressae sunt, et earum intellectus revelatus in mente».

75 «Quae non habent imagines sui similes» (Agostino, De Genesi ad litteram, XII, vi, 15).

76 Cfr. la ricostruzione di Rodolfi, Cognitio obumbrata.

77 «In visione autem spiritali, id est in corporum similitudinibus, quae spiritu videntur, fallitur anima, cum ea quae sic videt, ipsa corpora esse arbitratur; vel quod sibi suspicione falsaque coniectura finxerit, hoc etiam in corporibus putat, quae non visa coniectat. At vero in illis intellectualibus visis non fallitur: aut enim intellegit, et verum est; aut si verum non est, non 
nella vacanza papale, i secondi orgogliosi di aver contribuito alla drammatica situazione. Allo stesso tempo viene correttamente attribuita ai diffusori di profezie l'insistenza sulla "necessità" di un momento di tribolazione per la Chiesa, allo scopo di una futura rigenerazione. Possiamo anche individuare con buona approssimazione l'obiettivo della polemica dantesca in quei particolari testi, come le profezie papali figurate intitolate Genus nequam (la "stirpe cattiva"), che trasmettevano una serie di figure papali, affiancate da un motto e un commento: le prime figure erano identificate, a fondamento della veridicità della predizione; seguiva un gruppo di figure riferite ai o al futuro pontefice. Proprio intorno al 1304-1305 un codice fiorentino testimonia dell'uso di queste profezie in ambienti di tipo francescano o comunque tra gruppi nostalgici del papa Celestino $\mathrm{V}^{78}$ Che Dante conosca questa letteratura è confortato dalla immagine dei papi simoniaci di Inf. XIX, dove, come ha dimostrato Potestà, egli civetta esplicitamente con questa testualità. ${ }^{79}$

L'Alighieri, dunque, qui non sta mettendo in guardia dalla diffusione incontrollata di profezie, come fa ad esempio il teologo francescano Pietro di Giovanni Olivi: quest'ultimo, infatti, si trova a essere ispiratore, nel quadro di una rivalutazione della teologia della storia di impianto gioachimita, di gruppi che facevano grande uso di testi profetici, in particolare incentrati sulla denuncia dell'attuale stato della Chiesa. ${ }^{80}$ Risultava molto importante, di conseguenza, distinguere le corrette interpretazioni di visioni e avvenimenti contemporanei da quelle scorrette o equivoche. Dante, invece, sembra contestare in toto una visione che considera la vacanza papale - protratta a causa del cattivo uso del libero arbitrio da parte dei cardinali - come un momento necessario di svolta nella storia della salvezza (che può aprire, per esempio, all'arrivo di figure escatologiche come il papa angelico?).

Questa distinzione e precisazione è particolarmente importante a questa altezza cronologica del percorso dantesco. Nell'epistola XI, vero capolavoro del corpus, Dante è ormai giunto a un'esplicita autocoscienza profetica, basata su una assimilazione al profeta veterotestamentario Geremia. ${ }^{81}$ Egli sta sviluppan-

intellegit: unde aliud est in his errare quae videt, aliud ideo errare quia non videt» (Agostino, De Genesi ad litteram, XII, xxv, 52).

78 Si tratta di Firenze, Biblioteca Riccardiana, 1222 B, un codicetto dalla fattura piuttosto rozza, la cui redazione è stata realizzata poco dopo la morte di Benedetto XI; sul testo Genus nequam vedi Potestà, L'ultimo messia, p. 174.

79 Potestà, Dante profeta, pp. 85-87.

80 Burr, Olivi on Prophecy.

81 Cfr., in questo volume, il contributo di Potestà, "Cum Ieremia». Sul testo della lettera di Dante ai cardinali; sull'uso di Geremia in vista della investitura profetica, cfr. anche Jacoff, Dante, Geremia, e Tavoni, Qualche idea, pp. 161-ss. 
do un quadro cognitivo che gli consentirà di definire una propria ecclesiologia, nella visione del carro che chiude il Purgatorio e nella prospettiva di riforma disegnata nella Monarchia. Allo stesso tempo, e per la prima volta, il ruolo di denunciator è rivendicato, come si è già detto, proprio a partire da una posizione priva di auctoritas pastorale («nulla pastorali auctoritate»), quindi di laico nonché di povero («divitie mecum non sunt», Ep. XI, 9). L'assenza di legittimità istituzionale è finalmente non soltanto controbilanciata dal prestigio della scrittura volgare e dalla condizione di esule, ${ }^{82}$ ma viene finalmente superata da un progetto di legittimazione carismatica, per usare le categorie di Weber, ${ }^{83}$ derivata direttamente da Dio («gratia Dei sum id quod sum»), che si esplica nella missione apocalittica, affidata a Giovanni, di scrivere ciò che si vede: «quod vides scribe in libro» (Apc 1, 11).

82 Si tratta di una risorsa sfruttata da Dante nella lunga durata, per esempio anche in Tre donne; per le strategie di legittimazione di Dante durante l'esilio, cfr. Brilli, Firenze e il profeta. 83 Mi riferisco al celebre saggio del 1919 Politik als Beruf, che si può leggere in traduzione in Weber, La scienza come professione. 
